\title{
Facial Pressure Ulcers in a COVID-19 50-year-old Female Intubated Patient
}

\author{
Enrico M. Zingarelli ${ }^{1}$ Marco Ghiglione ${ }^{1} \quad$ Marianna Pesce $^{1} \quad$ lleana Orejuela ${ }^{1} \quad$ Silvia Scarrone ${ }^{2}$ \\ Renzo Panizza ${ }^{1}$
}

${ }^{1}$ Department of Plastic and Reconstructive Surgery,

SS.Antonio e Biagio e Cesare Arrigo Hospital, Alessandria, Italy

2Operating Theatre Management, SS.Antonio e Biagio e Cesare

Arrigo Hospital, Alessandria, Italy

\begin{abstract}
Address for correspondence Enrico M. Zingarelli, MD, Department of Plastic and Reconstructive Surgery, SS.Antonio e Biagio e Cesare Arrigo Hospital, Via Venezia 16, 15121 Alessandria, Italy (e-mail: enrico.zingarelli@gmail.com).
\end{abstract}

Indian J Plast Surg 2020;53:144-146

\begin{abstract}
Keywords

- SARS-CoV-2

- Covid-19

- facial pressure ulcers

In case of severe acute respiratory distress syndrome (ARDS), as in coronavirus disease 2019 (COVID-19) affected patients, the invasive ventilation in prone position can improve the prognosis, albeit with an increased risk of facial pressure ulcers. In this report, we will relate a case of facial pressure ulcers in a SARS-CoV-2 positive 50 yearold woman with the aim to describe the high-complexity management of COVID 19 in which healthcare professionals across the world are still involved.
\end{abstract}

\section{Introduction}

The worldwide diffusion of coronavirus disease 2019 (COVID-19) caused by severe acute respiratory syndrome coronavirus 2 (SARS-CoV-2) is characterized by various clinical presentations and different related complications.

Controlling the airways often requires mechanical invasive ventilation, ${ }^{1}$ and in cases of severe acute respiratory distress syndrome (ARDS), prone positioning of the patient can reduce mortality ${ }^{2}$ At the same time, however, the prone position ventilation can increase the risk of facial pressure ulcers. ${ }^{3}$

We report a case of facial pressure ulcers in a 50-year-old female, mechanically ventilated in a prone position, who was hospitalized for COVID-19.

The aim of this article is to contribute a little to better define the treatment complexities of the COVID-19 pandemic, in which healthcare professionals across the world are still involved.

\section{Case Report}

On March 10, 2020, a suspected 50 years-old female SARS-CoV-2 positive patient was admitted to the Emergency Department of the SS. Antonio and Biagio and C. Arrigo Hospital.

Clinical signs were fever, asthenia, and dry cough. In the previous 3 days, the patient had taken acetaminophen and antibiotics (amoxicillin and clavulanic acid $1 \mathrm{~g}$ three times per day), as prescribed by the practitioner. The patient also experienced diarrhea and cramp-like pain for 2 days before being admitted.

The patient's medical history was irrelevant, and no allergies were reported.

Vital parameters at the admission were: body temperature (BT) $38.8^{\circ} \mathrm{C}$, blood pressure (BP) $120 / 65 \mathrm{~mm} \mathrm{Hg}$, heart rate (HR) $95 / \mathrm{min}$, and respiratory rate 20/min. Pulse oximeter saturation was $87 \%$.

The physical examination was aspecific.

The laboratory findings revealed lymphopenya (lymphocytes $0.47 \times 1000 / \mathrm{mcl}$ ), elevated LDH (943 U/l), AST $(75 \mathrm{U} / \mathrm{l})$, ALT $(200 \mathrm{U} / \mathrm{l})$, GGT (41 U/l), procalcitonin (0.89 ng/mL), PCR (9.37), fibrinogen (596 mg/dl), and D dimer $(1.00 \mathrm{mcg} / \mathrm{mL})$.

With regard to radiological examinations, a chest CT showed peripheral consolidations and ground-glass opacities in both lungs. The SARS-CoV-2 positive nasopharyngeal swab confirmed the suspected diagnosis.

The patient was promptly intubated and transferred to a single room of the Intensive Care Unit of our hospital; ventilation was applied in a prone position.

After 15 days of intensive care, a plastic surgery evaluation was requested for multiple facial skin lesions. We observed pressure ulcers and skin lesions due to maceration involving
DOI https://doi.org/

10.1055/s-0040-1710403

ISSN 0970-0358.
License terms

(ㅇ) (1) $\ominus \circledast$ 


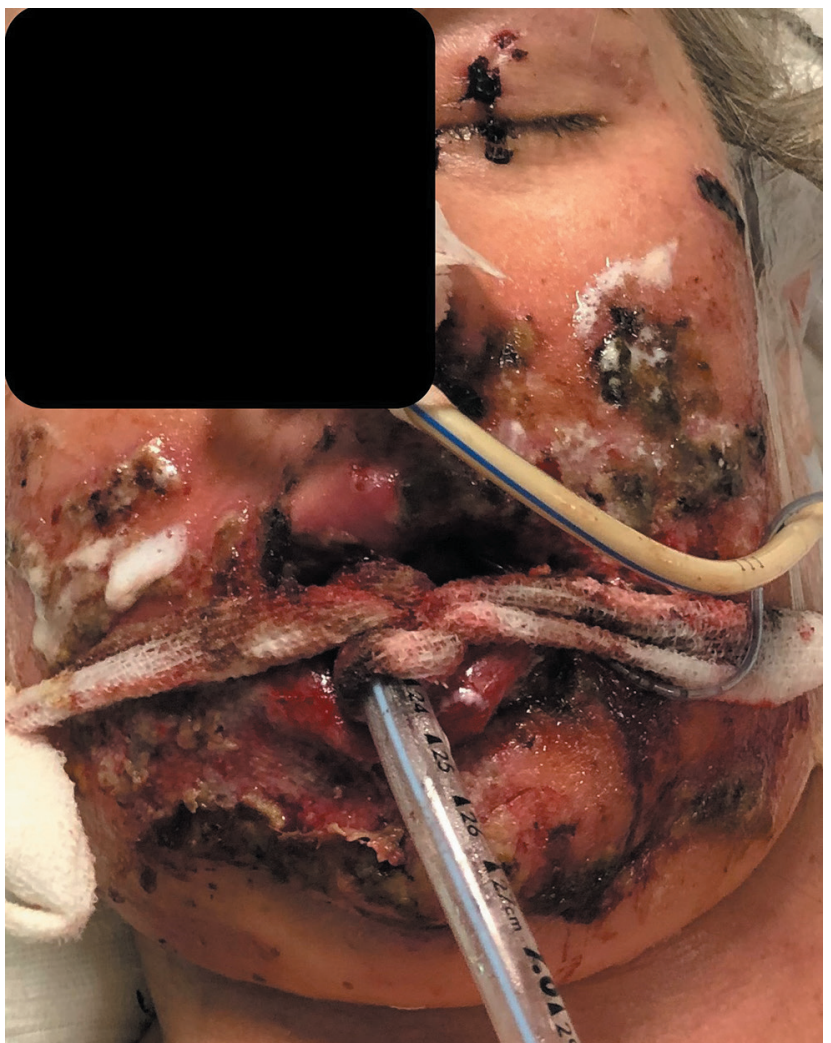

Fig. 1 Facial skin lesions after 15 days prone ventilation.

the lips, chin, perioral aerea, both cheeks, left zygomatic region, and superior and inferior left eyelids ( - Fig. 1).

The patient was evaluated by using all the required droplet and contact precautions. The sequence for putting on and taking off of personal protective equipment (PPE) was respected. Some pictures of the clinical case were taken by using a camera previously covered with adhesive film. As far as the dressing was concerned, we suggested the application of topical $1 \%$ Silver Sulfadiazine (Sofargen-Sofar), covering the wounds with gauzes impregnated with $0.2 \%$ Hyaluronic Acid And Sodium Salt (Connettivina-Fidia Farmaceutici s.p.a) and sterile gauzes. On the necrotic tissue on the eyelids and left zygomatic area, our dressing prescription was hyaluronic acid sodium salt collagenase ointment (Bionect Start-Fidia Farmaceutici s.p.a) twice per day.

One week after the first plastic surgery evaluation, the patient clinical conditions worsened and a tracheostomy was performed. However, skin lesions improved (-Fig. 2). At the time of the article's submission, the patient is still hospitalized.

\section{Discussion}

As reported by the Italian Ministry of Health, on April 2 at $5 \mathrm{pm}$ in Italy, there are 85388 SARS-CoV- 2 positive patients, 4068 of whom have been currently hospitalized in intensive care units in Italy, and 452 in Piedmont. Given the aggressive pulmonary involvement associated with

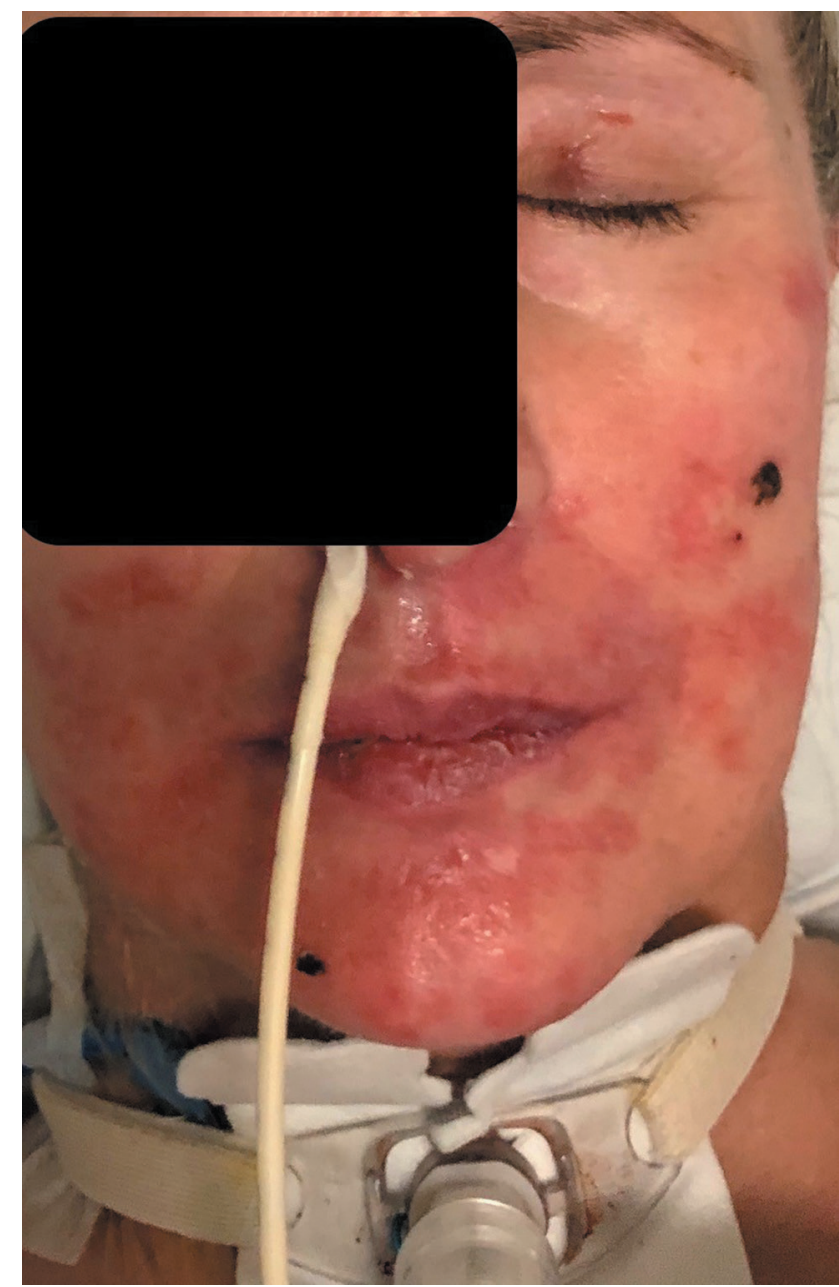

Fig. 2 Outcome after topical treatment.

COVID-19, noninvasive or invasive oxygen therapy is often required. On the basis of the potential risk of viral aerosolisation and the need for careful isolation precautions, noninvasive ventilation may be insufficient to manage COVID-19 induced respiratory failure. ${ }^{1}$ Moreover, there may be a poor response to noninvasive ventilation. ${ }^{1}$ Invasive ventilation is associated with reduced aerosolisation and is thus considered safer for staff and other patients. ${ }^{1}$

As reported in the literature, prone ventilation is likely to reduce mortality among patients with severe ARDS when applied for at least 12 hours daily. ${ }^{2}$ However, the prone position increases the risk of medical device-related pressure ulcers in the facial area. ${ }^{3}$

Despite the great effort made by all the healthcare professionals involved in the COVID 19 pandemic management, pressure ulcer preventive measures should be implemented. As suggested in the literature, a thin silicone foam dressing can represent a valid precaution approach. ${ }^{4}$ The frequent mobilization of the patients is mandatory to reduce the risk of facial pressure ulcers and avoid any cicatricial or permanent discromic effect. In this case, the suggested topical treatment promoted wound healing. 


\section{Conclusion}

This is the first report regarding facial pressure ulcer in COVID-19 affected patients. Considering the high number of hospitalized patients in intensive care units, it is of paramount importance to be aware of all the COVID-19 related complications with the aim to avoid possible discomfort of patients.

\section{Conflicts of Interest}

The authors declare no conflict of interest.

\section{Aknowledgments}

The authors wish to thanks all their colleagues for their unconditional devotion to manage the SARS-CoV-2 outbreak. We also acknowledge all the colleagues currently quarantined, following testing positive for COVID-19.

Thanks to Nicholas Cooper for the free article revision (e-mail: cooperenglishservices@gmail.com).

\section{References}

1 Sorbello M, El-Boghdadly K, Di Giacinto I, et al; Società Italiana di Anestesia Analgesia Rianimazione e Terapia Intensiva (SIAARTI) Airway Research Group, and The European Airway Management Society. The Italian coronavirus disease 2019 outbreak: recommendations from clinical practice. Anaesthesia 2020;(March):1-9

2 Munshi L, Del Sorbo L, Adhikari NKJ, et al. Prone position for acute respiratory distress syndrome: A systematic review and meta-analysis. Ann Am Thorac Soc 2017;14(October) (Supplement_4):S280-S288

3 Girard R, Baboi L, Ayzac L, Richard JC, Guérin C; Proseva trial group. The impact of patient positioning on pressure ulcers in patients with severe ARDS: results from a multicentre randomised controlled trial on prone positioning. Intensive Care Med 2014;40(3):397-403

4 Kim RS, Mullins K. Preventing facial pressure ulcers in acute respiratory distress syndrome (ARDS) J Wound Ostomy Continence Nurs 2016;43(4):427-429 